\title{
Revisiting the Compartment Fire
}

\author{
JOSÉ L. TORERO ${ }^{1}$, AGUSTIN H. MAJDALANI ${ }^{2}$, CECILIA ABECASSIS-EMPIS ${ }^{2}$ AND ADAM \\ COWLARD ${ }^{2}$ \\ ${ }^{1}$ School of Civil Engineering \\ The University of Queensland \\ Australia \\ ${ }^{2}$ BRE Centre for Fire Safety Engineering \\ The University of Edinburgh \\ United Kingdom
}

e-mail: J.Torero@uq.edu.au

\begin{abstract}
Understanding the relevant behaviour of fire in buildings is critical for the continued provision of fire safety solutions as infrastructure continually evolves. Traditionally, new and improved understanding has helped define more accurate classifications and correspondingly, better prescriptive solutions. Among all the different concepts emerging from research into fire behaviour, the "compartment fire" is probably the one that has most influenced the evolution of the built environment. Initially, compartmentalization was exploited as a means of reducing the rate of fire spread in buildings. Through the observations acquired in fires, it was concluded that reducing spread rates enabled safe egress and a more effective intervention by the fire service. Thus, different forms of compartmentalization permeated through most prescriptive codes. Once fire behaviour within a compartment was conceptualized on the basis of scientific principles, the "compartment fire" framework became a means to establish, under certain specific circumstances, temperatures and thermal loads imposed by a fire to a building. This resulted not only in improved codes but also in a scientifically based methodology for the assessment of structural performance. The last three decades have however seen an evolution of the built environment away from compartmentalization while the "compartment fire" framework has remained. It is therefore necessary to revisit the knowledge underpinning this seminal approach to initiate discussion of its continued relevance and applicability to an increasingly non-compartmentalised built environment. This paper, through a review of classic literature and the description of some recent experimentation, aims to inform and encourage such discussion.
\end{abstract}

KEYWORDS: compartment fires; fire dynamics; design fires.

\section{INTRODUCTION}

Predicting the progression of a fire is inevitably the starting step of any form of performance based design. Defining the time evolution of all relevant variables (temperatures, velocities, species, etc.) is the initial challenge that a Fire Engineer faces when attempting to quantify the performance of a system and all countermeasures introduced to mitigate the impact of a fire. Decades of research have focused on the different processes linked to the characterization of a fire environment and numerous engineering methods have been developed to enable engineers to quantify the performance of a design. This is particularly true when focusing on the evolution of a fire within buildings.

The evolution of a fire within a building is characterized by the coupling between the building and the combustion process. The environment resulting from the interaction of building and combustion can then be used to establish its influence on egress, countermeasures (detection and suppression) or on structural behaviour. Each component of a fire safety strategy is drastically different from the others therefore the evaluation of the performance of each component needs to be done in a manner consistent with the specific processes involved. A comprehensive assessment of the evolution of a fire and its interactions with people and buildings has long been recognized as an intractable problem. And while in recent years complex computational tools have been developed for all aspects of a fire strategy, it is still necessary, for the purpose of design, to develop simplified methods that allows an effective but manageable design process.

A common mechanism to simplify the design process is to separate the different components of the problem by linking specific processes to specific characteristic times within a fire timeline. For example, the characteristic timescales associated to egress and the activation of countermeasures are relatively small, 
therefore the assessment of their performance will emphasize the understanding of the earlier phases of a fire, i.e. fire growth time and time to flashover. In contrast, when addressing structural behaviour, growth and flashover occur within time scales that are much smaller than those required to significantly affect the mechanical strength of structural systems, thus the focus has been on fully developed fires. While a similar analysis could be done for each process, this paper focuses on the interaction between a fire and the structure. The simplified framework of a fully developed fire will be used to initiate the discussion. That being said, it is important to accept that this is already a simplification that excludes scenarios where the concept of a fully developed fire is not relevant.

The understanding of the thermal interaction between a fire and a structure has been explored since the late $19^{\text {th }}$ century. Bisby et al [1] provide a comprehensive review of the associated literature. The main achievement of the earlier stages of research was the definition of the standard temperature $v s$ time curve as a general description of the fire environment [2]. This definition enabled structural engineers to establish methods to protect structural systems from a fire. The recognition that further understanding of the fire environment is necessary to quantify structural behaviour only emerges through 30 years of research encompassing the period from 1960-1990. In this period, a series of seminal studies authored by the fathers of fire safety science provided the foundations for our current engineering methods. Kawagoe questions the physical basis of the standard temperature $v s$ time curve and is the first to intuitively establish the concept of the compartment fire [3]. Through experimental observations he defines the link between ventilation, gas phase temperatures and burning rate. Thomas and co-workers [4, 5] extend and formalize the experimental database into a series of engineering expressions that characterize the maximum temperature within a compartment and, given a fuel load, the potential duration of the a fully developed fire. Thomas's formulation de-emphasizes time and provides a worst case time invariant temperature regime for the fire until total burn-out of the fuel. At this point research bifurcates, while Petterson et al [6] extend the empirical data base by re-emphasizing the time evolution of the fire, Emmons et al [7] and McCaffrey et al [8] refine Thomas's formulation by further describing the different processes and adding more experimental data. It is important to note that only Petterson et al [6] emphasize the time evolution of the fire while all others focus on the worst case condition. Of notable importance are the studies by Harmathy et al [9], Law et al [10-12] and Tanaka et al [13-17] who attempted to translate the acquired knowledge into design methodologies with an emphasis on structural performance. Numerous complementary studies were conducted in this period providing refinements and extensions to the existing methods but emphasizing the validity of the fundamental approach initiated by Kawagoe [3].

In 1998 the SFPE Task Group on Fire Exposures to Structural Elements chaired by Prof. J.G. Quintiere started to develop the SFPE Engineering Guide for Fire Exposure to Structural Elements that was finally published in 2004 [18]. This guide provides a comprehensive review of the different methods used in the calculation of how fires thermally affect structures. This guide was followed in 2011 with the SFPE Engineering Standard on Calculating Fire Exposures to Structures [19] developed by SFPE StandardsMaking Committee on Calculating Fire Exposures to Structures chaired by J. K. Richardson. The SFPE Engineering Standard draws on the information of SFPE Engineering Guide to provide a method that enables the engineer to establish the evolution of the thermal boundary condition for a structure subject to a fire. Given a well-defined set of boundary conditions, the evolution of the transient temperature distribution of a structure can be established by means of a heat conduction analysis [4, 5, 9, 19-23]. These temperature distributions are then used as inputs for a structural analysis that determines the performance of a structural system in fire [23, 24]. There are other methods available in the literature [25-29] nevertheless, the comprehensive nature of the SFPE Guide and Standard make it a good starting point for this paper.

In parallel to the development of design methods, significant advances in the development of Computational Fluid Dynamics (CFD) models for fire applications have been reported. These models allow a significant level of refinement that enables a much more detailed treatment of the thermal boundary conditions for the structure. Successful applications have populated the literature in the last 10 years [30, 31]. CFD has a fundamental role in enabling better understanding of the physical processes [32] but it is recognized that there are still many uncertainties in the models. In what concerns the use of CFD for design, the utilization of the models can be too complex to be practical for main stream design and the drastic differences between solid and gas phase time scales do not necessarily justify the level of precision 
brought by the utilization of CFD [33]. This paper will not address further the use of CFD, this is a subject of enough complexity to merit its own individual work.

It could appear as if by 2011, date of the publication of the SFPE Engineering Standard on Calculating Fire Exposures to Structures [19], that research had matured to a level where the thermal boundary condition for a structure was fully defined. Existing design methodologies, albeit conservative, provided a robust solution to the thermal boundary condition for performance assessment of a structure in fire.

A review of the classical studies on which existing design methodologies are based serves to clarify the limitations of existing design methods and the areas where further research is necessary. Some new experiments will be presented to provide evidence towards the need for further research.

\section{THE COMPARTMENT FIRE FRAMEWWORK}

The temperature evolution within a building enclosure is defined by a compendium of complex processes occurring simultaneously. Fuel is pyrolyzed at a rate determined by the characteristics of the material and the net heat exchange between the fuel, the fire, the enclosure, the exterior environment and gas phase (hot and cold). The fuel mixes with oxidizer flowing through the compartment leading to a combustion reaction whose characteristics are defined by the relative quantities of fuel and oxidizer (local stoichiometry) as well by heat exchange with the enclosure and the exterior environment. The heat generated by the combustion reaction is partially lost at the openings, partially transferred to the enclosure and to a minor extent fed back to the fuel. The relative importance of all these terms defines the energy accumulated in the compartment and thus its temperature evolution. The resulting gas phase temperature will most likely be a function of all three spatial coordinates and time $\left(\mathrm{T}_{\mathrm{g}}(\mathrm{x}, \mathrm{y}, \mathrm{z}, \mathrm{t})\right)$ and the consequence of complex heat and mass transfer processes. The characteristic time scales of combustion, flow and heat transfer can be very different, thus significant simplifications are potentially possible. Given the complexity of the processes an a priori assessment of the possible simplifications is not possible without a detailed quantification of each term.

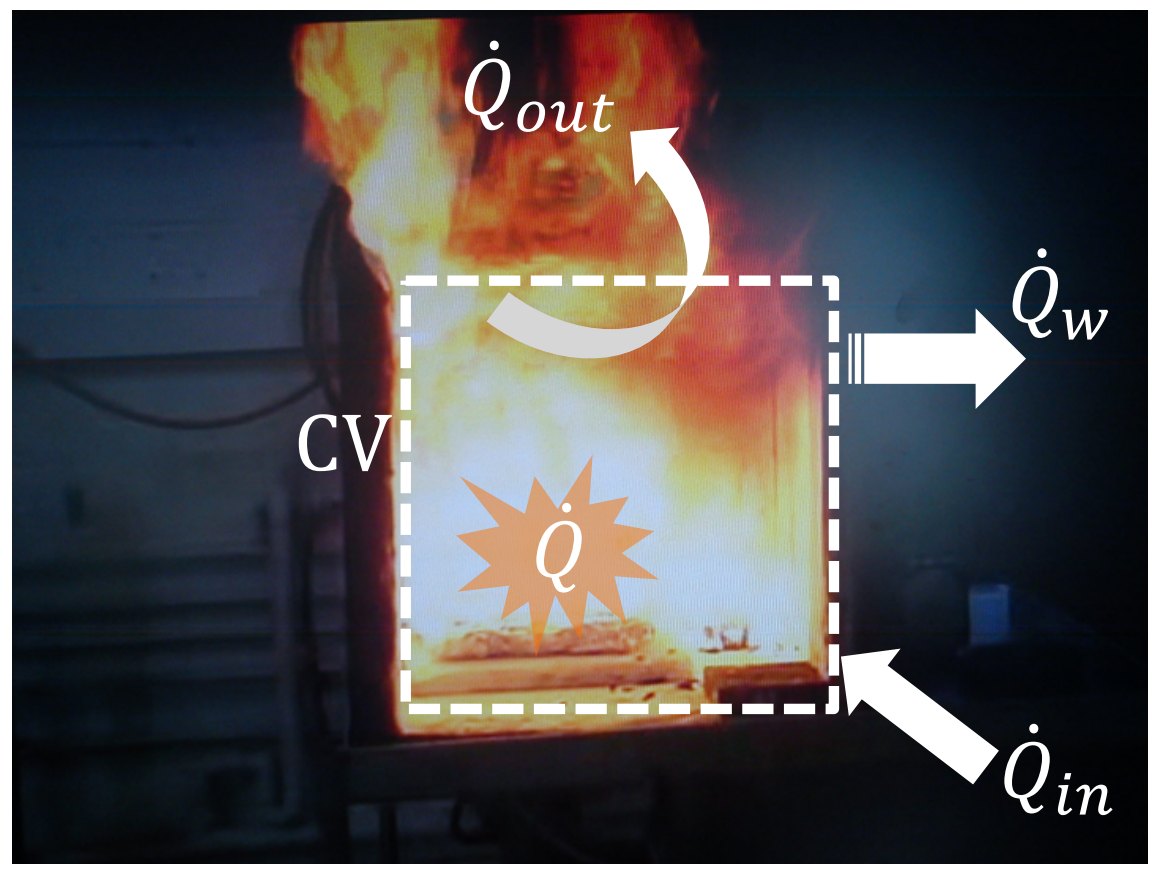

Fig. 1. The "Compartment Fire" framework.

The "Compartment Fire" framework is based on a series of observations that emerged from numerous experiments conducted by Kawagoe [3] and later by Thomas et al. [5]. These experiments were then complemented by results of Harmathy [9]. The principle behind the method is that characteristic time scales for combustion are very short thus energy is assumed to be released as a function of reactant supply, 
i.e. oxygen in the case of an oxygen-limited reaction and fuel in the case of a fuel-limited reaction. The characteristic time for heating of the enclosure is extremely long compared to all gas phase processes, therefore the gas phase can be assumed to be quasi-steady. The characteristic time for fuel pyrolysis is comparable to that of solid heating therefore a single characteristic time describes both. The burning rate will attain steady state conditions at the same time as the enclosure reaches thermal equilibrium with the gas phase. Under those circumstances a maximum gas phase temperature will be achieved (and maintained) beyond the transient heating of the enclosure. The steady state condition implies a constant heat transfer to the walls, a constant generation of heat, and a constant flow of heat out of the enclosure. The main consequence of this approach is that the geometry of the enclosure (dimensions, aspect ratio and openings) defines the relative importance of each term and therefore the value of the equilibrium gas phase temperature $\left(\mathrm{T}_{\mathrm{g}, \max }(\mathrm{x}, \mathrm{y}, \mathrm{z})\right)$. Thomas explores all three parameter spaces emphasizing the role of each of them $[4,5]$.

As explained above, depending on the availability of air the generation of heat is either controlled by fuel supply (fuel limited) or air supply (oxygen limited). This distinction is important in that it determines the form the energy source takes when introduced in the energy equation. Nevertheless, it does not eliminate the need to resolve the transport processes that bring fuel and oxidizer towards the reaction zone. Thomas et al. [4] describe the role of transport by establishing two limit regimes, Regime I and Regime II. Harmathy [9], offers a similar discussion using a different terminology ("ventilation controlled," "fuel-surfacecontrolled") but identical concepts.

Thomas et al. [4] describe Regime I as the case where the vents are small enough that they allow for the compartment to fill with smoke. In this case, at steady-state oxygen supply is limited, combustion is rich and soot concentrations are high. A significant amount of the energy released by combustion occurs outside the compartment and the flow field within the enclosure is dominated by thermal expansion of the gases allowing the assumption that momentum within the enclosure is negligible. Momentum and mass is only exchanged at the openings therefore it can be characterized by static pressure differentials across the openings. If a fixed fraction of the energy released is lost through the openings then the maximum temperature distribution $\left(\mathrm{T}_{\mathrm{g}, \max }(\mathrm{x}, \mathrm{y}, \mathrm{z})\right)$ will only be a function of the equilibrium heat exchange with the enclosure and the heat generated, where the heat generated is directly related to the mass flow of oxygen through the vents. Given the high soot concentrations (the optical depth is very small) and low velocities a linearized approximation for total heat transfer is acceptable. This enables not only the formulation of heat exchange through the walls by simple expressions $[18,33]$ but also the expression of the burning rate as a direct function of the gas phase temperature. As Thomas et al. [4] and Harmathy [9] point out, the equilibrium temperature, and consequently all other characteristic values, are defined by the relative magnitude of the three main terms of the energy equation, heat generation, heat transfer to the enclosure and heat losses through the vents. The relative values are therefore strongly dependent on the geometry of the compartment. While the validity of the framework extends to all geometries that comply with the assumptions, the resulting values are defined by the complex heat and mass transfer processes that remained unresolved. All unresolved processes are substituted by experimental values therefore the quantitative values extracted from experimentation are only applicable for the characteristic geometries reported in the tests.

In the case where the vents are sufficiently large, the smoke evacuates the enclosure with little resistance allowing for the fire to draw air. If the pressure differentials generated by the fire dominate over the static pressure differentials, the combustion products are expelled from the enclosure as fast as air is drawn into the enclosure. Complex heat and mass transfer processes dominate over this regime that Thomas et al. [4] label Regime II. No simple theoretical analysis can be defined for Regime II. Characteristic heat transfer times are short, soot concentrations are low therefore heat exchange from the fire to the structure was deemed to be less severe than for Regime I [4]. Harmathy [20] argues theoretically the lower severity of Regime II showing that the large velocities and vent size result in a major fraction of the heat being expelled through the vents, a decreased net heat accumulation in the enclosure and lower gas phase temperatures. Quantification of the actual heat transfer to the structure and the fuel is highly dependent on the geometry of the enclosure and extrapolation is extremely difficult under these circumstances. If quantification of the environment is desired, Regime II needs to be avoided. 
From a design perspective different approaches can be followed. Code based restrictions on vent and compartment size can be imposed to avoid either regime. Through Thomas's work it is generally implied that Regime I is more severe than Regime II (albeit never truly quantified) therefore building design can be done under the quantifiable worst case conditions of Regime I. In other words, the building geometry can be restricted so that only Regime I fires are possible and structural design can be done to withstand the thermal load of such fires. Alternatively, an even more conservative approach can be followed which is to quantify Regime I as a worst case condition but require building characteristics consistent with Regime II. A final approach was strongly advocated by Harmathy [20-22] who embraced Thomas's conclusion on the greater severity of Regime I and encouraged designers to increase venting as an effective means to reduce fire proofing. The following quote is extracted from Reference [21]: "The simplest way of improving fire safety is to reduce its destructive potential in the "fire cell" (space on fire) by ensuring that the fire, if it occurs, will be fuel-surface-controlled (i.e. Regime II), in other words, by using large window areas, whenever possible, it becomes possible to replace fire resistance requirements with ventilation requirements. This means that the designer is entitled to decide whether to choose between buildings built with small windows and heavy fire-rated walls and floors, and buildings with large windows and lighter non-combustible, non-fire-rated elements." Whatever approach is followed, it was recognized that this is only valid within the context of the specific geometries studied [5, 9].

An important point regarding the differences between Regime I and Regime II is highlighted by Harmathy [20]. The mechanisms linking compartment temperature and burning rate are only valid for Regime I and not for Regime II, in particular, the inverse relationship between the maximum average gas phase temperature $\left(T_{\mathrm{g}, \max }\right)$ and the duration of the fire. In Reference [20] Harmathy indicates: "The conclusion reached so far is that well-ventilated fires, i.e., fuel-surface controlled fires, not only burn at lower temperatures (in general), but also are very short. The common belief that compartment fires are either short and hot or long and relatively cool is, therefore, completely wrong." This observation seems to have been forgotten and it has become common to describe both regimes as being defined by the same interaction of physical processes [34] without remembering that Regimes I and II are two limit forms of behaviour that are the result of neglecting different processes and having the remaining ones interacting in a very specific manner.

In summary, the "Compartment Fire" framework is a robust representation of the behaviour of a fire in an enclosure. There is no fundamental weakness in the approach but the quantitative results are intimately linked to the geometry of the compartment (size, vent size, aspect ratio). The geometry will define if the conditions are consistent with the assumptions of the analysis but most importantly, it will establish the relative magnitude of the heat flow in and out of the enclosure (terms in Figure 1), which in turn defines the equilibrium temperature. Currently, the relative distribution is not defined in an analytic way but by means of experimental values. Extrapolation of these experimental values requires geometrical consistency. The SFPE Engineering Guide for Fire Exposure to Structural Elements [18] addresses this issue as early as the Executive Summary, nevertheless it addresses the influence of the geometry on the validity of the different methodologies employed in terms that are relevant only to Regime I fires, in other words as a function of the Opening Factor $\left(A / A_{0} \sqrt{H_{0}}\right)$. The following section will define this terminology and address the fundamental differences between both regimes.

\section{Energy Balance in a Compartment Fire}

A compartment will be used as a control volume to describe the mechanisms by which energy can be transferred in and out of the control volume resulting in temperature distributions within the compartment. Friction work will be neglected and the volume of the compartment will be assumed constant. The energy conservation equation for the fixed control volume of Figure 1 can be represented as:

$\frac{d Q_{C V}}{d t}=\dot{Q}_{\text {in }}-\dot{Q}_{\text {out }}+\dot{Q}-\dot{Q}_{w}$

Where $\dot{Q}_{\text {in }}$ is the enthalpy entering the control volume with the reactants per unit time, $\dot{Q}_{\text {out }}$ is the enthalpy leaving the control volume with the products per unit time, $\dot{Q}_{w}$ represents the heat losses to the enclosure 
boundaries and $\dot{Q}$ the total heat release rate within the enclosure by the fire. To understand better the role of each term it is useful to normalize the Equation (1) by the heat release rate.

$\frac{1}{\dot{Q}} \frac{d Q_{C V}}{d t}=1+\frac{\dot{Q}_{\text {in }}}{\dot{Q}}-\frac{\dot{Q}_{\text {out }}}{\dot{Q}}-\frac{\dot{Q}_{w}}{\dot{Q}}$

Given that the addition of mass into the enclosure associated to the fuel's mass loss rate is negligible in comparison to the air mass inflow and gas mass outflow rates (estimated between $16 \%$ [9] and $18 \%$ [4]), the rate of change of mass within the control volume can be considered close to zero (i.e. $\frac{d m_{C V}}{d t} \approx 0$ ), therefore the transient term of the conservation of mass equation can be neglected resulting in:

$\dot{m}_{\text {in }}=\dot{m}_{\text {out }}=\dot{m}$

Given the significant temperature differences between the reactants and products it is possible to establish that $\frac{\dot{Q}_{\text {in }}}{\dot{Q}} \ll \frac{\dot{Q}_{\text {out }}}{\dot{Q}}$. This simplification is not necessary for the rest of the analysis but it will be retained here for consistency with the original presentation. Simple scaling analysis establishes that the characteristic heating times for the solid walls is at least two orders of magnitude longer than that of the combustion products allowing to assume quasi-steady conditions, i.e. $\frac{1}{\dot{Q}} \frac{d \dot{Q}_{C V}}{d t} \approx 0$. On the basis of these simplifications equation (3) can be rewritten as

$1=\frac{\dot{Q}_{\text {out }}}{\dot{Q}}+\frac{\dot{Q}_{w}}{\dot{Q}}$

It is important to note that until this point no strong assumptions have been made and Equation (4) can be satisfied by any enclosure subject to a fire. What follows is a series of assumptions that enable the transformation of Equation (4) into a set of very simple expressions that serve to characterize the enclosure fire under Regime I conditions. The assumptions are:

(a) The heat release rate is defined by the complete consumption of all oxygen entering the compartment and its subsequent transformation into energy, $\dot{Q}=\dot{m} Y_{\mathrm{O}_{2}, \infty} \Delta H c_{\mathrm{O}_{2}}$. Where the ambient oxygen concentration is given by $Y_{O_{2}, \infty}$ and the heat of combustion per kilogram of oxygen consumed is given by $\Delta \mathrm{Hc}_{\mathrm{O}_{2}}$. This assumption not only eliminates the need to define the oxygen concentration in the outgoing combustion products but also eliminates the need to resolve the oxygen transport equation within the compartment. Implicitly this assumption limits the analysis to scenarios where there is excess fuel availability, chemistry is fast enough to consume all oxygen transported to the reaction zone and the control volume acts as a perfectly stirred reactor. It is important to add that if the heat of combustion is assumed to be an invariant then the level of completeness of the combustion process is assumed to be independent of the compartment.

(b) Radiative losses through the openings are assumed to be negligible [4] therefore $\dot{Q}_{\text {out }}$ is treated as an advection term. Harmathy [9] provides an estimate for the radiative losses of approximately $3 \%$ of the total energy released.

(c) There are no gas or solid phase temperature spatial distributions within the compartment. The gas phase equilibrium temperature is therefore defined by a single value, $T_{g, \max }$, and the equilibrium surface temperature of all solid surfaces also by a single value, $\mathrm{T}_{\mathrm{w}} . \quad \dot{Q}_{\text {out }}$ and $\dot{Q}_{w}$ can then be strongly simplified. If the specific heat is assumed to be a constant then $\dot{Q}_{o u t}=\dot{m} C_{p} T_{g, \text { max }} . \dot{Q}_{w}$ 
can be simplified in several manners depending on the objectives of the simplification. The simplifications associated to $\dot{Q}_{w}$ require more detail and are addressed later.

(d) Mass transfer through the openings is governed by static pressure differences therefore a simple orifice plate expression can be used to evaluate the mass flow of air through the openings, $\dot{m}=C A_{O} \sqrt{H_{O}}$, where $A_{O}$ and $H_{O}$ are the opening area and height respectively and $\mathrm{C}$ is a constant that amalgamates all other constants including the orifice plate coefficient and gravity. It is important to note that this assumption requires all velocities within the compartment to be negligible. Different values of the constant were derived by Harmathy [9] and calculated by Thomas [5] for different experimental conditions.

The classical approach is to define $\dot{Q}_{w}$ as conduction losses through the boundaries of the compartment. While more complex formulations are possible, a simple steady state approximation will be used to quantify conductive losses:

$\dot{Q}_{W}=A k \frac{\left(T_{g, \max }-T_{\infty}\right)}{\delta}$

Where $A$ is the area through which heat is being transferred, $k$ an effective thermal conductivity of the compartment boundaries, $\delta$ a characteristic thickness of the boundaries and $T_{\infty}$ the ambient temperature. It is important to note that this approximation is quite coarse in that it assumes the temperature difference between the interior and the exterior of the compartment boundaries $\left(T_{g, \max }-T_{\infty}\right)$ as the maximum possible value i.e. $T_{\infty}$ does not rise due to heat transfer through the walls, therefore the resulting heat transfer to the boundaries is maximized and the compartment temperature is minimized as a consequence of these maximal heat losses. While this approximation might not be conservative it is useful to establish the relationship between the gas phase temperature, the air intake and the compartment geometry. Substituting equation (5) into equation (4) and solving for the steady state gas phase temperature the following expression is obtained:

$T_{g, \text { max }}=\left(\frac{1+\frac{T_{\infty}}{T_{C D}}}{1+\frac{T_{F}}{T_{C D}}}\right) T_{F}$

Where $T_{F}=Y_{O_{2, \infty}} \Delta H c_{O_{2}} / C_{p}$ is a characteristic flame temperature and $T_{C D}=\frac{C Y_{O_{2, \infty}} \Delta H c_{O_{2}}}{(k / \delta)}\left(\frac{A_{O} \sqrt{H_{O}}}{A}\right)$ is a characteristic conduction temperature. It is important to note that under the present assumptions all terms of equation (6) are constant with the exception of the classic opening factor $\left(\frac{A_{O} \sqrt{H_{O}}}{A}\right)$ and the thermal conduction heat transfer coefficient $(k / \delta)$ both properties of the compartment.

An alternative approach to define the heat transfer through the walls is by means of a convective boundary condition. In this case heat transfer through the walls can be described by

$\dot{Q}_{W}=A h_{T}\left(T_{g, \max }-T_{W}\right)$

Where $h_{T}$ is a total heat transfer coefficient and $T_{W}$ the interior surface temperature of the compartment boundaries. Once again, this is a very simple expression that establishes a different form of the steady state gas phase temperature: 
$T_{g, \text { max }}=\left(\frac{1+\frac{T_{W}}{T_{C V}}}{1+\frac{T_{F}}{T_{C V}}}\right) T_{F}$

Where $T_{C V}=\frac{C Y_{O_{2, \infty}} \Delta \mathrm{HCO}_{2}}{h_{T}}\left(\frac{A_{O} \sqrt{H_{O}}}{A}\right)$ is a characteristic convection temperature. While both expressions (equations (6) and (8)) are very similar and depend on the opening factor $\left(\frac{A_{O} \sqrt{H_{O}}}{A}\right)$, equation (8) also depends on a gas phase parameter which is the total heat transfer coefficient. This only becomes interesting when the asymptotic conditions are attained.

If $\frac{T_{\infty}}{T_{C D}} \ll 1$ and $\frac{T_{F}}{T_{C D}} \ll 1$ (i.e. large opening factor and insulating walls) then equation (6) results in

$T_{g, \max }=T_{F}$

If $\frac{T_{\infty}}{T_{C D}} \gg 1$ and $\frac{T_{F}}{T_{C D}} \gg 1$ (i.e. small opening factor and non-insulating walls) then equation (6) results in

$T_{g, \max }=T_{\infty}$

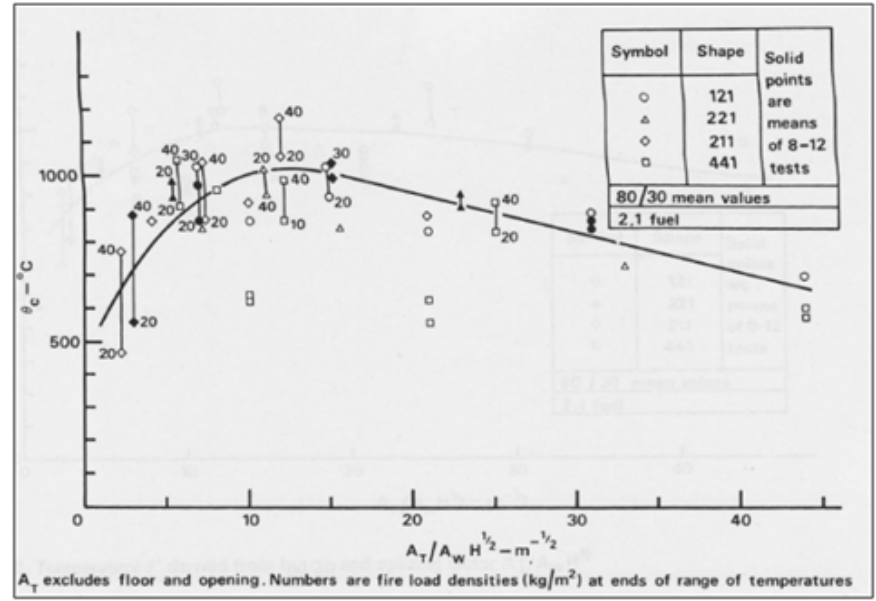

Fig. 2. Average compartment temperature for the CIB Tests, extracted from [5].

Similarly, if $\frac{T_{\infty}}{T_{C V}} \ll 1$ and $\frac{T_{F}}{T_{C V}} \ll 1$ (i.e. very large opening factor and weak total heat transfer) then equation (8) results in

$T_{g, \max }=T_{F}$

But if $\frac{T_{\infty}}{T_{C V}} \gg 1$ and $\frac{T_{F}}{T_{C V}} \gg 1$ (i.e. very small opening factor and strong total heat transfer) then equation (8) results in

$T_{g, \max }=T_{W}$ 
It is important to note that the asymptotic values associated with equation (6) (i.e. equations (9) and (10)) directly relate the gas phase temperature to the two hard limits, the ambient and characteristic flame temperatures. Therefore, this equation is very useful when addressing the evolution of the gas phase temperature as a function of the opening factor. In the work by Thomas et al [4] it is stated that the quantitative values of $T_{g, \max }$ will be dependent on the conduction heat transfer coefficient $(k / \delta)$ and that a conservative characteristic value can be taken for testing leading to a conservative empirical evolution of $T_{g, \max }$ as a function of only the opening factor. Figure 2 shows the plot extracted from reference [5] where the right hand side of the curve shows the evolution of $T_{g, \max }$ as a function of the inverse of the opening factor. Extrapolation of the trend in this region in both directions will lead towards the asymptotic values defined by equations (9) and (10). The asymptotic limit defined by equation (9) will not be attained. Towards the left of the maximum temperature (Figure 5) the conditions are representative of Regime II which does not comply with the assumptions of this analysis and therefore deviates from the trends defined by equation (6) and the asymptotic limit defined by equation (9).

Finally, Kawagoe [3] establishes that from equation (5) and from the fact that the burning rate is proportional to the heat transfer rate to any of the boundaries of the compartment, that the burning rate is proportional to $A_{O} \sqrt{H_{O}}$.

In contrast to equation (6), equation (8) has a different asymptotic term for the case where heat transfer between the gas and compartment is high. In this case the gas phase and wall temperatures are the same (as represented by equation (12)). This observation is significant in that it focuses on the temperature of the structure and indicates that given the right heat transfer conditions, the thermal characteristics of the structure can dominate the minimum temperature of the gas phase and the balance of the two right hand terms of equation (4).

In summary, the compartment fire framework allows, by means of several strong assumptions, a representation of the maximum steady state temperature, $T_{g, \max }$, of a compartment simply as a function of the opening factor $\left(\frac{A_{O} \sqrt{H_{O}}}{A}\right)$ and the burning rate, $R$, as proportional to the ventilation factor $A_{O} \sqrt{H_{O}}$. Those assumptions are consistent with Regime $I$, and while remaining within these assumptions, simple expressions (equations (6) and (8)) can be used to link $T_{g, \max }$ to the opening factor. Outside the validity of the fundamental assumptions the theoretical link between temperature, burning rate and opening factor does not exist.

\section{THE RANGE OF VALIDITY OF THE COMPARTMENT FIRE FRAMEWWORK}

\section{The Original Experiments}

To understand the range of validity of the expressions presented above it is important to discuss the experiments that led to this analysis. Two series of large scale experiments provide the initial set of data used to develop the Compartment Fire framework: those by Kawagoe [3] and those of the CIB study [4, 5]. The CIB programme summarizes Kawagoe's experiments therefore will be used to describe the nature of the tests. The shape of the compartments used in the CIB Programme was rectangular, designated by a three figure code representing the three principal dimensions of width, depth and height (where all dimensions are normalized by the height). Thus, a 211 compartment measured 2 units wide, 1 unit depth and 1 unit height. The four shapes of compartment examined were $211,121,221$, and 441 . The overall scale of the compartment was taken as the compartment height, and scales of $0.5,1$, and 1.5 meter were employed. Therefore, the larger compartment size was $6 \mathrm{~m}$ x $6 \mathrm{~m}$ x $1.5 \mathrm{~m}$ height.

The data obtained through these experiments are mainly burning rates and average compartment temperatures $\left(T_{g, \max }\right)$. The weight of the fuel was obtained throughout each test either by weighing the whole compartment or by weighing the floor separately. The temperatures were recorded by only two thermocouples placed at $1 / 4$ and $3 / 4$ of the compartment height above the centre of the floor. As pointed out by Thomas and Heselden [5], in some cases, the lower thermocouple was laid inside the wood cribs that were used as fuel resulting in a measurement bias for the average temperature.

Although the CIB data for average temperatures vs. the inverse opening factor presented significant scatter, Thomas and Heselden [5] drew a best-fit line through one of the fuel configuration data sets (i.e. the $(2,1)$ 
crib configuration, meaning $20 \mathrm{~mm}$ thick sticks spaced $20 \mathrm{~mm}$ apart) obtaining as a result the very wellknown plot presented in Figure 2.

Similar graphs for other fuel crib configurations were presented by Thomas and Heselden [5] but because the $(2,1)$ configuration resulted in higher average temperatures, the data of Figure 2 are generally used for design analysis. It is important to emphasize that the data shows reasonable scatter for Regime I conditions but the scatter is very large for Regime II. In the case of Regime II, factors such as aspect ratio, nature of the fuel and scale were shown by the authors to have a significant effect on the resulting temperatures.

An important finding in the CIB Programme was that high values of the enclosure's depth to height ratio produced non-uniform temperatures horizontally and large windows non-uniformity vertically, with the ceiling temperatures typically being the maximum temperatures found in an enclosure fire.

\section{Non-Uniformity and the "Natural Fire"}

Following the observations of the CIB studies, Stern-Gottfried et al. [35] reviews the test literature in an attempt to establish if the assumption of homogeneous temperature distribution within the compartment is valid through the available data. The compiled data showed that for smaller, cubic compartments the assumption of a homogeneous temperature distribution is valid but it breaks down with the size of the compartment and in particular when the aspect ratio deviates from the cubic compartment.

Additionally, Drysdale [36] explains that most of our knowledge of the behaviour of compartment fires comes from experiments with near-cubical compartments, with characteristic dimensions ranging from 0.5 $\mathrm{m}$ to $3 \mathrm{~m}$ which of course are very different in shape and size compared with typical spaces in modern commercial buildings [37].

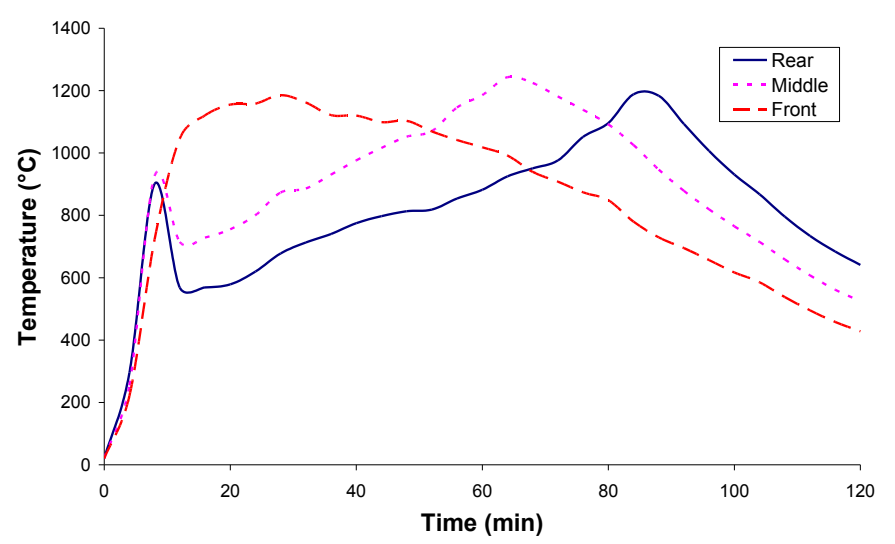

Fig. 3. Temperature distributions for the Natural Fire safety Concept Tests [29]

In 1999, the Natural Fire Safety Concept 2 test series at Cardington [38] included a much greater spatial resolution of instrumentation. The eight Cardington tests were conducted in a room $12 \mathrm{~m}$ x $12 \mathrm{~m}$ x $3 \mathrm{~m}$ with uniformly spaced fuel load packages distributed across the floor. Sixteen thermocouple trees containing four thermocouples each were placed on a uniform grid in the compartment to record the gas temperatures, shown in Figure 3. The tests were conducted with various combinations of fuel type, ventilation distribution, and interior lining material. The tests had liquid fuel channels connecting the fuel packages so that ignition and the subsequent burning could be as uniform as possible.

The Cardington experiments intended to test two types of compartment insulation; "insulating" and "highly insulating". However, after Test 1, the "highly insulating" material was placed on the ceiling for all remaining tests, creating an intermediate level of insulation. The fuel packages were either just wood cribs or a combination of wood and plastic cribs. The ventilation openings were either fully open on the front of the enclosure or fully open on the front and back. As shown in Figure 3 there is a significant evolution in space and time of the temperature. The evolution in time is mostly related to the time lag between heating in the gas phase and the heating of the compartment, thus is related to the level of insulation. This had been 
previously extensively studied by Pettersson et al [6] when developing the parametric fire curves, nevertheless the Natural Fire Concept tests were the first where the insulation was systematically varied. The evolution in space was observed but not studied in detail due to the restricted amount of instrumentation.

A different form of temperature distribution was reviewed and reported by Clifton [39] who describes fires that spread through a large compartment generating spatial and temporal distributions as a consequence not only of stratification but also of the progression of the fire through the compartment. Clifton [39] emphasizes a simple methodology to model these fires and only presents a limited set of experimental data to validate the analytical approach. While the data are coarse and limited, it does indicate drastic spatial and temperature evolutions throughout the compartment. An earlier study concerning the St. Lawrence Burn project [40] was recently reported by Gales [41] where compartments of dimensions $11.2 \mathrm{~m} \mathrm{x} 12.8 \mathrm{~m}$ and $13 \mathrm{~m} \times 9 \mathrm{~m}$ respectively were exposed to a propagating fire showing once again significant spatial and temporal distributions.

\section{The Dalmarnock Fire Tests}

The Dalmarnock Fire Tests, which provide the greatest instrumentation density to date, were conducted in a real high-rise apartment building in Glasgow, UK [42]. The two tests conducted had a realistic fuel load of typical furnishings. The compartment was $4.75 \mathrm{~m} \times 3.50 \mathrm{~m} \times 2.45 \mathrm{~m}$, containing 20 thermocouple trees, each with 12 thermocouples (placed $0,0.05,0.1,0.2,0.3,0.4,0.6,0.8,1.0,1.3,1.6$ and $2 \mathrm{~m}$ from the ceiling). Ignition occurred in the waste-paper basket adjacent to the sofa. Two tests were conducted, Test One was allowed to progress to burn-out while the second test was manually suppressed immediately after flashover. Thus only Test One is of interest here.

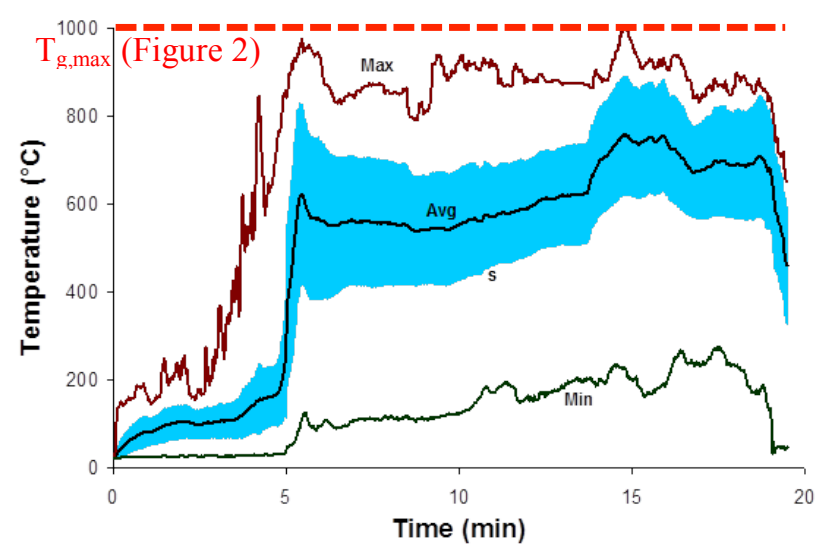

Fig. 4. Experimental results of Dalmarnock Test One [42] showing the compartment average, maximum and minimum temperatures, the standard deviation and the prediction from Figure 2. Flashover occurred at $5 \mathrm{~min}$ and the fully developed stage lasted until suppression at $19 \mathrm{~min}$.

The Dalmarnock Test One shows that the temperature distributions, even for a small compartment corresponding to Regime I, are lower than the predictions of the CIB and present very large variations in space and time. All it takes is to provide sufficient instrumentation density to spatially resolve the temperature fields. In the Natural Fire Concept tests the compartment was large but all fires were ignited simultaneously, in the Dalmarnock tests the fire was allowed to propagate, but the compartment was small. In the study by Clifton [39] one series of experiments was reported where the compartment was large (44 $\mathrm{m}$ $\mathrm{x} 34 \mathrm{~m}$ ) and the fire was allowed to propagate, nevertheless, the data has poor resolution and only a single experimental condition is reported. A similar situation occurs with the St. Lawrence Burns Project where only two tests were reported and limited data is available.

\section{Summary of Existing Evidence}

The available experimental data confirm that the observations and analysis first developed by Kawagoe [3] and then by Thomas et al $[4,5]$ can provide a simple yet fundamentally adequate way to describe the 
maximum average temperature, $T_{g, \max }$, and burning rate as a function of a single parameter, the ventilation factor $\left(\frac{A_{O} \sqrt{H_{O}}}{A}\right)$. The SFPE Engineering Guide - Fire Exposures to Structural Elements [18] reviews a large amount of data that validates this approach. The data and analysis is presented in the context of the of the same methodology and while it explores the limits of validity of the approach it does not extended beyond the "Compartment Fire Framework" first formulated by Thomas et al [4]. Following on from Thomas et al [4], this paper highlights the main assumptions involved in the formulation that leads to the definition of the ventilation factor $\left(\frac{A_{O} \sqrt{H_{O}}}{A}\right)$ as the single parameter that characterizes the compartment fire and establishes through a brief review of some experimental data that there is a large body of evidence that demonstrates that the conditions necessary for a Regime I fire are not necessarily met in modern building enclosures. Furthermore, the available data is not sufficient to characterize the different forms of behaviour that fires can have in the multiplicity of compartment geometries now common in the built environment. The next section briefly summarizes a series of tests designed to start filling these gaps of knowledge.

\section{THE "TALL BUILDING" TESTS}

To respond to the need to better describe the special and temporal evolution of a fire that is allowed to progress in a large compartment a series of tests were conducted in 2013 at the Building Research Establishment in the UK. These tests are inscribed within a larger programme that looks to address fire scenarios for tall buildings, thus will be labelled the "Tall Building Tests."

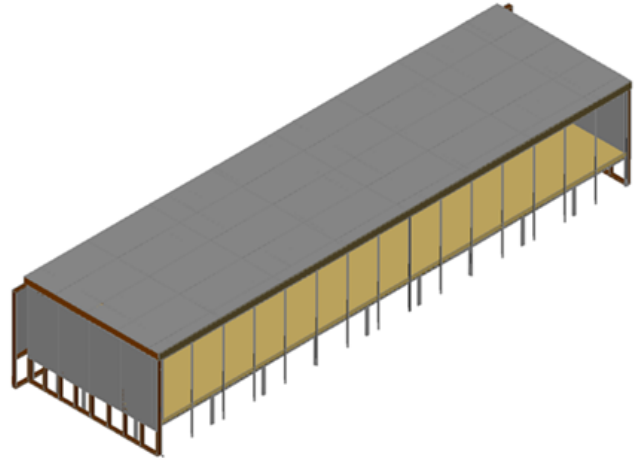

(a)

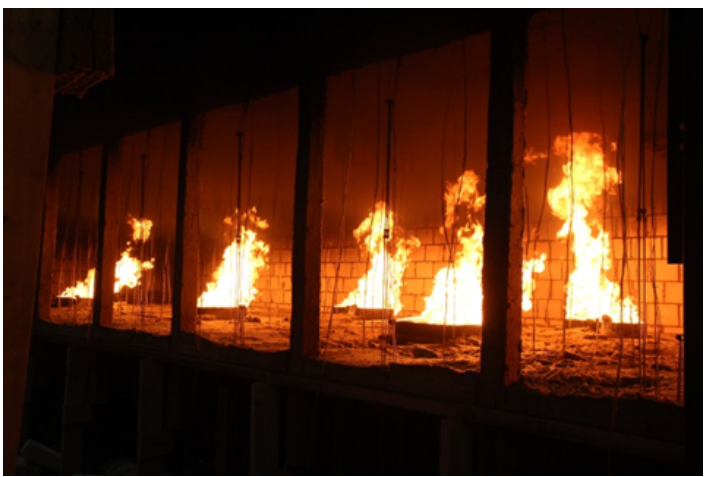

(b)

Fig. 5. (a) Schematic of the BRE large compartment constructed and (b), a photograph of a characteristic gas burner test.

A compartment of dimensions $5 \mathrm{~m} \times 18 \mathrm{~m} \times 2 \mathrm{~m}$ (Figure 5(a)) was constructed and included 15 openings along the front $(1.5 \mathrm{~m}$ high by about $1 \mathrm{~m}$ wide each) that can be open and closed to allow varying ventilation in a systematic way. The tests are heavily instrumented including internal thermocouple trees spaced at $0.7 \mathrm{~m}$ in the $\mathrm{x}$-direction, $0.6 \mathrm{~m}$ in the $\mathrm{y}$-direction, and at $0.3,0.6,0.9,1.2,1.4,1.6,1.8$, and $1.95 \mathrm{~m}$ in the z-direction. There is also a thermocouple tree in the centre of each opening at $\mathrm{z}=0.25,0.5,0.75,1.0$, and $1.25 \mathrm{~m}$. Outside there is also a thermocouple tree at around $0.4 \mathrm{~m}$ from the compartment and aligned with the centre of each opening. These trees have 12 thermocouples each which are spaced as follows: $0.25,0.5,0.75,1.0,1.25,1.5,1.75,2.0,2.25,2.5,2.75$ and $3.0 \mathrm{~m} .100$ thermocouples provide in-depth temperatures (at different depths) along a $3 \mathrm{~m}$ wide section of the back wall which is in-filled with nonflammable insulation. Heat flux gauges were placed on all 5 surfaces of the compartment evenly distributed. There are 45 on the floor ( 3 in $\mathrm{x}$-direction, 15 in y-direction), 45 on the ceiling, 45 along the back wall (15 in y-direction, 3 in z-direction) and 15 (5 in the x-direction, 3 in the z-direction) along each of the side walls. There are also heat flux gauges outside, opposite the centre of each opening, at different distances away from the compartment (in x-direction). Smoke obscuration is measured with 5 laserreceiver pairs in total evenly spaced along the centre of the compartment. Bi-directional velocity probes allow characterizing the in-flow and out-flow of the compartment. There are 2 probes per opening $(\mathrm{z}=$ 
$0.225 \mathrm{~m}$ and $\mathrm{z}=1.275 \mathrm{~m}$ of the opening height, respectively). There are 5 gas-sampling points evenly spaced along the ceiling of the compartment. The gas probes sample $\mathrm{O}_{2}, \mathrm{CO}_{2}$ and $\mathrm{CO}$ to establish completeness of combustion. Five cameras were used to film the compartment from different angles, including one camera at the centre of each of the side walls. An InfraRed camera was also used to film the compartment from the outside.

$$
t=130 \mathrm{~s}, \mathrm{x}=1800 \mathrm{~mm}
$$

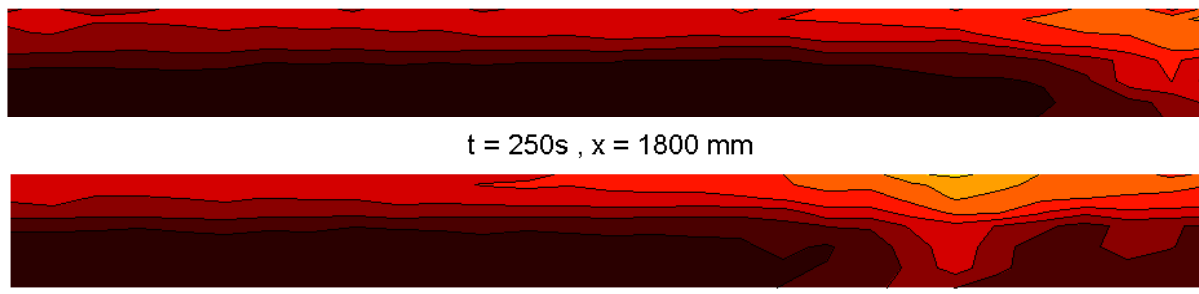

$$
\mathrm{t}=370 \mathrm{~s}, \mathrm{x}=1800 \mathrm{~mm}
$$

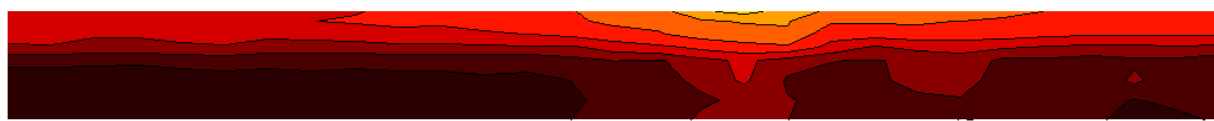

$$
\mathrm{t}=610 \mathrm{~s}, \mathrm{x}=1800 \mathrm{~mm}
$$

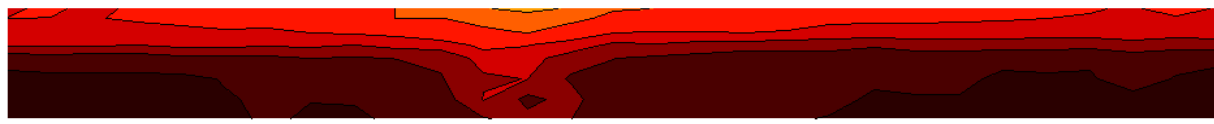

$$
\mathrm{t}=730 \mathrm{~s}, \mathrm{x}=1800 \mathrm{~mm}
$$
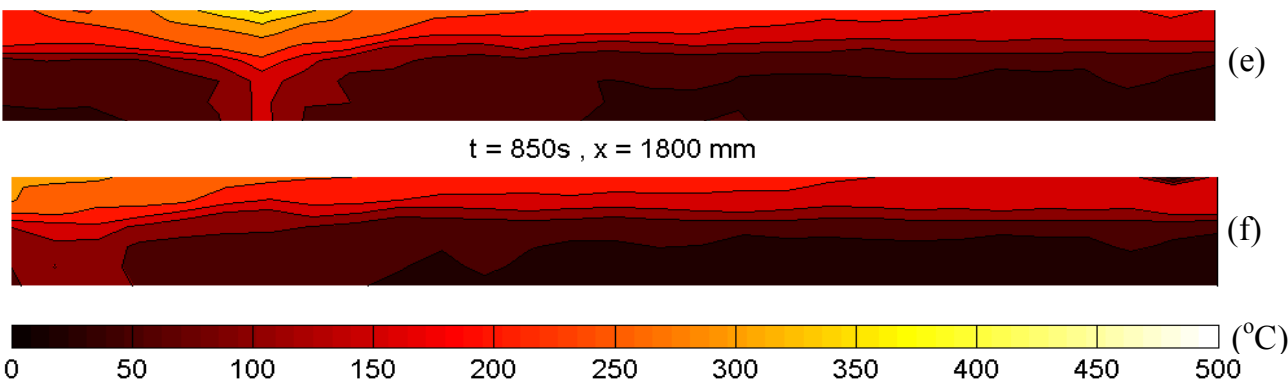

Fig. 6. Temperature distributions along a plane $1800 \mathrm{~mm}$ deep and parallel to the open face (in between burners) - the openings were fully open allowing for the maximum evacuation of of smoke.

Two series of tests were conducted, a first series where a sequence of gas burners ( 12 propane burners, of $0.5 \mathrm{~m} \times 0.5 \mathrm{~m}$ trays full of gravel, evenly spaced throughout the compartment in pairs of 2) was ignited progressing from one end of the compartment to the other. Ventilation and fire spread were varied to cover a range that allowed for spread much faster than ventilation opening to spread much slower than ventilation opening. The second series of tests was conducted with wood cribs covering the entire floor and ignited at one end. The ventilation was again varied. For the wood crib tests, the central staging area has been divided into 8 sections that can move up and down independently. These sat on load cell systems that enabled the measurement of mass loss. A photograph of a typical test is presented in Figure 5(b).

While the description of the experimental data is beyond the scope of this paper, it is important to emphasize that the scale and data variety and resolution result in different observed behaviour that not only deviate from the compartment fire framework but that could potentially have a significant impact on the thermal boundary condition used to analyse structural behaviour. Furthermore, current detailed structural analysis requires a level of resolution that cannot be provided by the simple formulation of the compartment fire framework $[34,44]$. These tests provide a level of resolution that is more consistent with the needs of such analysis. 
Figure 6 shows a series of representative data for an experiment using gas burners ignited in a sequential manner while the panels allowing air were opened fully. The fire is initiated with two burners (front and back -Figure 5(b)) at the right hand side of the compartment. Initially, the ceiling jet propagates across the compartment with no significant accumulation of smoke but very rapidly it covers the entire compartment $(<130 \mathrm{sec}-$ Figure 6(a)). The smoke produced by the fire is fully evacuated allowing for the establishment of steady state conditions. The initial burners are turned-off as the next set is ignited and the temperatures once again reach steady-state conditions rapidly. A clear smoke layer can be seen in Figure 6(b). The smoke layer shows very similar temperatures to the ones observed in Figure 6(a).

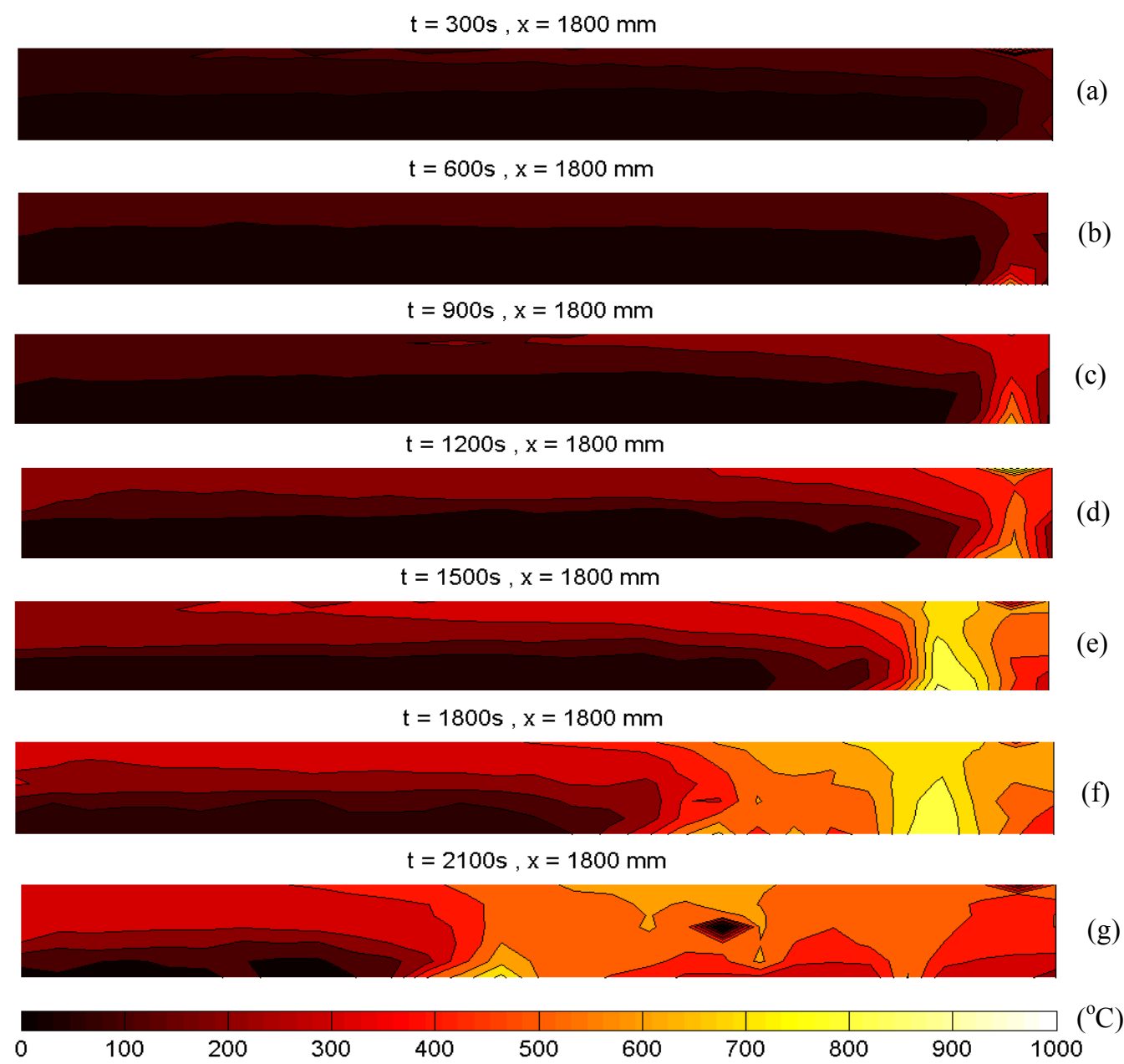

Fig. 7. Temperature distributions along a plane $1800 \mathrm{~mm}$ deep and parallel to the open face - the openings were fully open allowing for the maximum evacuation of of smoke and the fuel were wood cribs.

This series of tests shows at each stage conditions very similar to those described by Regime II. These conditions reproduce themselves at each stage of the burning process and the characteristic times scales in the gas phase are short enough that quasi-steady conditions can be established at each stage of the propagation. The burners were ignited at rates consistent with spread rates of typical building fuels, therefore these observations could potentially be extrapolated to fires with realistic materials. While the conditions are similar to those of Regime II fires, the size of the compartment allows for the formation of gradients of temperature not only in the vertical direction but also in the directions parallel to the floor. The data has enough resolution to be able to provide an appropriate boundary condition for structures whose analysis requires spatial distribution. 
The rate of ignition of the gas burners as well as the size and location of the openings were varied to establish other potential regimes. The rate of ignition of the burners as well as the size of the fire (i.e. number of burners) did not have a major impact on the nature of the fire in the compartment within the range of conditions studied. A clear smoke layer was rapidly established with the interface dependent mostly on the size of the fire indicating that the capacity of the open face to evacuate smoke exceeded the differences introduced by the changes to the fire. In contrast, as the vents were diminished in size or in number, smoke could not be evacuated and the quasi-steady nature of the process was lost leading to a complex and dynamic interaction between burners and smoke.

An experiment with wood cribs can be used as an example as it encompasses the full potential complexity of the dynamic interaction between the fire, the smoke and the compartment. Figure 7 presents temperature distributions within a plane (1800 $\mathrm{mm}$ deep) at different points in time. The fuel is wood cribs and the vents are fully open. The fire was ignited in the right hand corner and allowed to propagate. Initially the ceiling jet propagates across the compartment (Figure 7(a)) until a smoke layer is established (Figure 7(b)). Fire spread is very slow relative to the gas phase processes thus quasi-steady state conditions establish in a similar manner to those presented in Figure 6. As the fire continues to grow the temperature of the smoke layer starts to increase. An important aspect of this is that depending on the size of the fire and the amount of ventilation surface available, the rate at which conditions evolve in the vicinity of the fire is much different to the rate of evolution in the far field (left hand side Figure 7(c)). Furthermore, momentumdriven flows impinging on the walls start affecting the characteristics of the smoke layer (Figure 7(d)). At approximately 1500 second (Figure 7(e)) smoke layer temperatures on the right hand side of the compartment exceed $500^{\circ} \mathrm{C}$ within approximately a third of the compartment. At this stage rapid ignition of the fuel through almost half of the compartment occurs in a manner that resembles a localized flashover (Figure 7(f)). The fire will continue to burn to the right of the flame front (Figure 7(g)) but the burning rate is maximum at the leading edge of the flame decreasing towards the right of the compartment. For the case where the vents were fully open, the flames continue to spread towards the left of the compartment. Strong air entrainment from left to right and smoke evacuation behind the flame prevented any subsequent instantaneous ignition of the fuel.

The experimental sequence presented above is described only with the purpose of illustrating the complex dynamics of the fire within a large compartment. The different processes explained varied in their significance depending on the ventilation and it was very clear that the temperature distributions were a strong function of the geometry of the compartment. What is clear is that under these conditions the dynamics of the fire correspond to a complex mixture of the limit Regimes I and II described by Thomas et al [5] and there is no relationship between the overall opening factor $\left(\frac{A_{O} \sqrt{H_{O}}}{A}\right)$ and the temperatures or burning rates.

\section{SUMMARY}

Upon revisiting the original studies that define the compartment fire framework it is clear that the approach that links an averaged maximum steady state temperature, $T_{g, \max }$, and burning rate, $R$, to an opening factor $\left(\frac{A_{O} \sqrt{H_{O}}}{A}\right)$ and an air inflow parameter $\left(A_{O} \sqrt{H_{O}}\right)$ respectively, is a simple but robust way to describe the behaviour of a Regime $I$ fire. The conditions of a Regime $I$ fire are defined by a series of very strong assumptions that guarantee the direct link between ventilation, temperature and burning rate. There is no theoretical link between the opening factor and the gas phase temperature for Regime II fires and any experimental evidence of a link is accompanied by great scatter of the data. This is not a new observation, from the very early studies by Thomas et al, the scatter of the data within Regime II conditions was emphasized.

There is significant experimental data that shows conditions under which the assumptions of the compartment fire framework are not valid but there are no systematic studies that truly address the boundaries of validity of this approach. Therefore, the limits of validity of the methodology are currently unknown. 
A critical assumptions associated to the compartment fire framework is the geometry of the compartment. Most of the data that validates the method is with quasi-cubic small $\left(<150 \mathrm{~m}^{3}\right)$ enclosures. Recent experimental data on well instrumented fires in larger compartments has demonstrated complex behaviour that cannot be described in terms of the compartment fire framework. Many modern building spaces deviate from the small quasi-cubic enclosure therefore there is great need to conduct research that provides physical insight on the dynamics of a fire in complex geometries. High resolution data is necessary for validation of design tools intended to describe the thermal boundary condition for a structure within a fire in a large compartment.

\section{ACKNOWLEDGMENTS}

The authors wish to thank the many people involved in the large scale experiments; colleagues that have helped, through extensive intellectual discussions, to formulate the thoughts expressed in this paper and the many students involved in this work through the years. The commitment of BRE to this work has, in many ways made it possible, as well as the support from EPSRC and the many industrial partners that through the years have supported this work.

\section{REFERENCES}

[1] L. Bisby, J. Gales, and C. Maluk, "A contemporary review of large-scale non-standard structural fire testing," Fire Sci. Rev., vol. 2, no. 1, p. 1, 2013.

[2] Ingberg, S.H. "Fire loads," Quarterly Journal of The National Fire Protection Association, 22, 4361.," Quaterly J. NFPA, vol. 22, pp. 43-61, 1928.

[3] Kawagoe, K., "Fire Behaviour in Rooms - Report No. 27," 1958.

[4] Thomas, P.H., Heselden, A. J. and Law, M. "Fully-developed compartment fires -two kinds of behaviour," no. 18. 1967.

[5] Thomas, P.H., and Heselden, A.J.M., "Fully developed fires in single compartments", CIB Report No 20. Fire Research Note 923, Fire Research Station, Borehamwood, England, UK, 1972.

[6] Pettersson, O. Magnusson, S. E. and Thor, J. "Fire Engineering Design of Steel Structures," Stockholm, Jun. 1976.

[7] Prahl, J. and Emmons, H.W. "Fire-induced Flow Through an Opening," Combust. Flame, vol. 25, pp. 369-385, May 1975.

[8] McCaffrey, B.J. and Rockett, J.A., "Static Pressure Measurements of Enclosure Fires," J. Res. Natl. Bur. Stand. (1934)., vol. 82, no. 2, pp. 107-117, Sep. 1977.

[9] Harmathy, T.Z. “A New Look at Compartment Fires,” Fire Technolgy, vol. 8, no. 3, pp. 196-217, Aug. 1972.

[10] Law, M., “A Relationship between Fire Grading and Building Design and Contents - FRN No. 877," Borehamwood, 1971.

[11] Law, M., "Designing Fire Safety for Steel - Recent Work,” Proc. ASCE Spring Conv. ..., pp. 135$150,1981$.

[12] Law, M., “A Basis for The Design of Fire Protection of Building Structures,” Struct. Eng., no. February, pp. 25-33, 1983. 
[13] Tanaka, T., “A Mathematical Model of a Compartment Fire - BRI Research Paper No. 70,” Tokio, Japan, 1977.

[14] Tanaka, T., "A Model on Fire Spread in Small Scale Buildings - 1st Report - BRI Research Paper No.79," Tokio, Japan, 1978.

[15] Tanaka, T., "A Model on Fire Spread in Small Scale Buildings - 2nd Report - BRI Research Paper No. 84," Tokio, Japan, 1980.

[16] Tanaka, T., "A Performance Based Design Method for Fire Safety of Buildings - NISTIR 4449," 1990.

[17] Tanaka, T., "Simple Formula for Ventilation Controlled Fire Temperatures - NISTIR 6030," 1997.

[18] "Engineering Guide - Fire Exposures to Structural Elements," Society of Fire Protection Engineers, 2004.

[19] "Engineering Standard - Calculating Fire Exposures to Structures," Society of Fire Protection Engineers, 2011.

[20] Harmathy, T. Z., "Design of Buildings for Fire Safety - Part I," Fire Technol., vol. 12, no. 2, pp. 95-108, 1976.

[21] Harmathy, T.Z., "Design of Buildings for Life Safety Part II," Fire Technol., vol. 12, no. 3, pp. 219-236, 1976.

[22] Harmathy,T.Z., "Designers Option: Fire Resistance or Ventilation," in 6th CIB Congress - The Impact of Research on The Built Environment, 1974, no. 436, pp. 277-282.

[23] Buchanan, A., "The Challenges of Predicting Structural Performance in Fires," Fire Safety Science, 9: 79-90, 2008.

[24] Franssen, J.M., "Structures In Fire, Yesterday, Today And Tomorrow," Fire Safety Science, 8: 21$35,2005$.

[25] Law, M., "A Basis for the Design of Fire Protection of Building Structures," The Structural Engineer 61A:5 (January 1983).

[26] Lie, T.T., Structural Fire Protection, New York, American Society of Civil Engineers, 1992, pp. 137-158.

[27] "Fire Engineering Design Guide, Report of a Study Group of the New Zealand Structural Engineering Society and the New Zealand National Fire Protection Association," A. Buchanan, ed., 1994.

[28] Eurocode 1: Actions on structures - Part 1-2: General actions - Actions on structures exposed to fire, EN 1991-1-2, CEN Brussels, Nov. 2002.

[29] The SFPE Handbook of Fire Protection Engineering, $4^{\text {th }}$ Ed., Quincy, Mass., National Fire Protection Association, 2009. 
[30] Baum, H.R., "Simulating Fire Effects On Complex Building Structures," Fire Safety Science, 8: 3$18,2005$.

[31] McGrattan, K. B., "Fire Modeling: Where Are We? Where Are We Going?" Fire Safety Science, 8: $18-23,2005$.

[32] Rein,G., Zhang, X., Williams, P., Hume, B., Heise, A., Jowsey, A., Lane, B., Torero, J.L., "Multistory Fire Analysis for High-Rise Buildings," Proceedings of the $11^{\text {th }}$ International Interflam Conference, London, Sept. 2007.

[33] Jowsey, A., Welch, S. and Torero, J.L., "Heat and Mass Transfer for Modeling of Structures in Fire," Transport Phenomena in Fire, B. Sunden and M. Faghri Editors, WIT Press, UK, Chapter 4, 2007.

[34] Usmani, A.S., Rotter, J. M., Lamont, S., Sanad, A.M. and Gillie, M. Fundamental principles of structural behaviour under thermal effects", Fire Safety Journal, 36, 721-744, 2001.

[35] Stern-Gottfried, J., Rein, G., Bisby, L. and Torero, J.L., "Experimental review of the homogeneous temperature assumption in post-flashover compartment fires", Fire Safety Journal, 45 (2010) 249261.

[36] Drysdale, D., An Introduction to Fire Dynamics ( $3^{\text {rd }}$ edition), John Wiley and Sons, Chichester, 2011.

[37] Jonsdottir, A., Rein, G., "Out of Range (Travelling Fires),” Fire Risk Management, pp. 14-17, Dec 2009.

[38] Kirby, B.R., Wainman, D.E., Tomlinson, L.N., Kay, T.R. and Peacock, B.N., "Natural Fires in Large Scale Compartments" - A British Steel Fire Research Station Technical Collaborative Project. BSC, 1994.

[39] Clifton, G.C., "Fire Models for Large Fire cells," HERA Report R4-83, HERA, Manukau City, New Zealand, March 1996.

[40] Shorter G.W. "The St. Lawrence Burns General Report." National Research Council Canada, Research Report, DBR-IR-150, 1959.

[41] Gales, J., "Travelling Fires and the St. lawrence Burns Project," Fire Technology, November 2013.

[42] Abecassis-Empis, C., Reszka, P., Steinhaus, T., Cowlard, A., Biteau, H., Welch, S., Rein, G. and Torero, J.L. "Characterization of Dalmarnock fire test one," Experimental Thermal and Fluid Science, vol. 32, no. 7, pp. $1334-1343,2008$.

[43] Cowlard, A., Bittern, A., Abecassis-Empis,C. and Torero, J.L. "Some Considerations for the Fire Safe Design of Tall Buildings," International Journal of High-Rise Buildings, March, Vol 2, No 1, 2013. 